\title{
Detection of Left Arm and Left Leg Lead-wire Interchange Based on Serial ECGs
}

\author{
Richard E Gregg ${ }^{1}$, Saeed Babaeizadeh ${ }^{1}$ \\ ${ }^{1}$ Philips Healthcare, Cambridge, MA, USA
}

\begin{abstract}
Left arm and left leg lead-wire interchange (LA-LL) is difficult to detect because a normal ECG still appears normal with such interchange. The aim of this study is to design and evaluate a LA-LL interchange algorithm based on serial ECG differences.

A large database of ECGs $(n=146,000)$ was separated into serial ECG pairs for the patients with multiple ECGs. There were 89,600 patients and 25,700 had multiple ECGs. The average beats of serial ECG pairs, QT region only, were subtracted to construct a single 16 bit greyscale ECG image. The STT region was normalized for heart rate by a linear correction. The LA-LL positive set was created by simulated interchange in the current ECG. We split the ECG images randomly into training (70\%), test (20\%) and validation (10\%) subsets. With the goal of high specificity, class weights were biased toward low false positives for training.

The CNN sensitivity (SE) and specificity (SP) were $84.5 \%$ and $99.5 \%$ respectively. Assuming a prevalence of $L A-L L$ reversal of $0.5 \%$ in clinical practice, the estimated $P P V$ and $F 1$ were $46 \%$ and $60 \%$.

Detecting LA-LL reversal is the most challenging in common lead-wire interchanges. Compared to the Kors algorithm with SE/SP of 18/99.5\%, this algorithm shows a significant boost in sensitivity with the same specificity.
\end{abstract}

\section{Introduction}

Lead-wire interchange in diagnostic 12-lead ECG is a well-known quality issue for ECG recordings. The most common lead-wire interchange is the connection of the left arm wire to the right arm electrode and right arm wire to left electrode [1]. Interchanges can contribute to incorrect diagnosis. There are many ECG clinical case reports showing the problem ECG with an undetected lead-wire reversal, the misdiagnosis, and then the appearance of the ECG once repeated without the interchange. Greenfield found a left arm and left leg reversal led to a potential diagnosis of recent inferior myocardial infarction or possibly pulmonary embolism [2]. Bailey analysed inferior STEMI with LA-LL interchange and found consistent detection of lateral STEMI [3]. Karur found a LA-LL interchange mimicking new ST elevation in leads I and aVL [4].

In the process of developing lead reversal algorithms and database collection, various authors have estimated the prevalence. Hedén found that the arm lead reversal is the most common with other lead reversals following at lower levels according to Table 1 [1].

Table 1. Prevalence of common lead-wire interchanges

\begin{tabular}{ll}
\hline Lead-wire interchange & Prevalence $(\%)$ \\
\hline RA-LA & $0.43(47 / 11,009)$ \\
RA-LL & $0.29(32 / 11,009)$ \\
LA-LL & $0.07(8 / 11,009)$ \\
Chest lead, cumulative & $0.23(25 / 11,009)$ \\
Total & $1.0(112 / 11,009)$ \\
\hline
\end{tabular}

Several different approaches have been employed for automated detection of limb lead interchange and precordial lead interchange. Most algorithms treat within limb lead changes and within precordial lead changes. Those are the most common and easy to simulate. One algorithm approach is to exploit the redundancy in ECG since leads are highly correlated. A second main approach is machine learning using ECG measurements such as frontal plane axis and per lead P-wave amplitude. A third approach is to use an end to end machine learning solution with the ECG signal as input to a deep neural network. Kors and Feild independently built algorithms based on the redundancy between ECG leads as the basis for high performing algorithms $[5,6]$. Hedén used ECG features as input to a neural network to detect limb and precordial lead interchanges [1,7]. Han used both ECG lead correlation and ECG measurements as input to a machine learning approach [8].

The left arm / left leg (LA-LL) interchange is particularly difficult to detect both by expert readers and by algorithm. When looking at other algorithms, the difficulty is evident by the difference in performance of the LA-LL compared to other reversals. Kors found sensitivities between $87 \%$ and $99 \%$ for limb reversals but only $18 \%$ for LA-LL [6]. Hedén and Han did not publish numbers for LA-LL presumably because that interchange is so difficult to detect. 
In the development effort for lead reversal detection by Feild and later by Han, the ECG database was created from serial ECGs so that any true reversals could be excluded. Lead reversals were simulated only using ECGs free from lead-wire interchange. For that effort, all reversals were simulated. The use of serial ECG as a form of truth for lead reversal database development sparked the idea for the current study, detection using serial ECGs.

\section{Methods}

\subsection{Study database}

The study database consists of ECGs from patients with multiple ECGs in a large single-center database collected over a period of 3 years. There were 146,000 ECGs in the database, but only a subset of 25,700 patients (of 89,600 total) had multiple ECGs resulting in 56,582 ECG pairs. The test $(10 \%)$, training $(80 \%)$ and validation $(10 \%)$ datasets of ECG pairs were randomly assigned. The training and validation sets were used in algorithm development, but the hold out test was only used for testing once training was complete. Each ECG current/previous pair was used in the LA-LL positive and LA-LL negative sets. The LA-LL positive set was created by simulating the LA-LL interchange in the current ECG of each pair. The LA-LL interchange can be simulated by the operations indicated in Table 2 below.

Table 2. Mathematical operations to simulate the LA-LL lead-wire interchange.

\begin{tabular}{ll}
\hline Original lead & ...becomes \\
\hline I & II \\
II & I \\
III & -III \\
aVF & aVL \\
aVL & aVF \\
\hline
\end{tabular}

\subsection{Algorithm}

The algorithm for detection of left arm and left leg leadwire interchange was based on subtracting the average beats of the current and previous ECGs. Both current and previous ECGs were pre-processed to create a time aligned average beat to reduce the effect of artefact. To account for the difference in heart rates, the QT interval of the previous ECG was normalized to the heart rate difference with the current ECG using the Hodges heart rate correction for QT interval [9]. The Hodges correction is a linear correction which allows for proportional resampling across the interval from QRS end to end of T-wave. Figure 1 shows an example of a patient's previous and current ECG average beats with a simulation of the LA-LL interchange
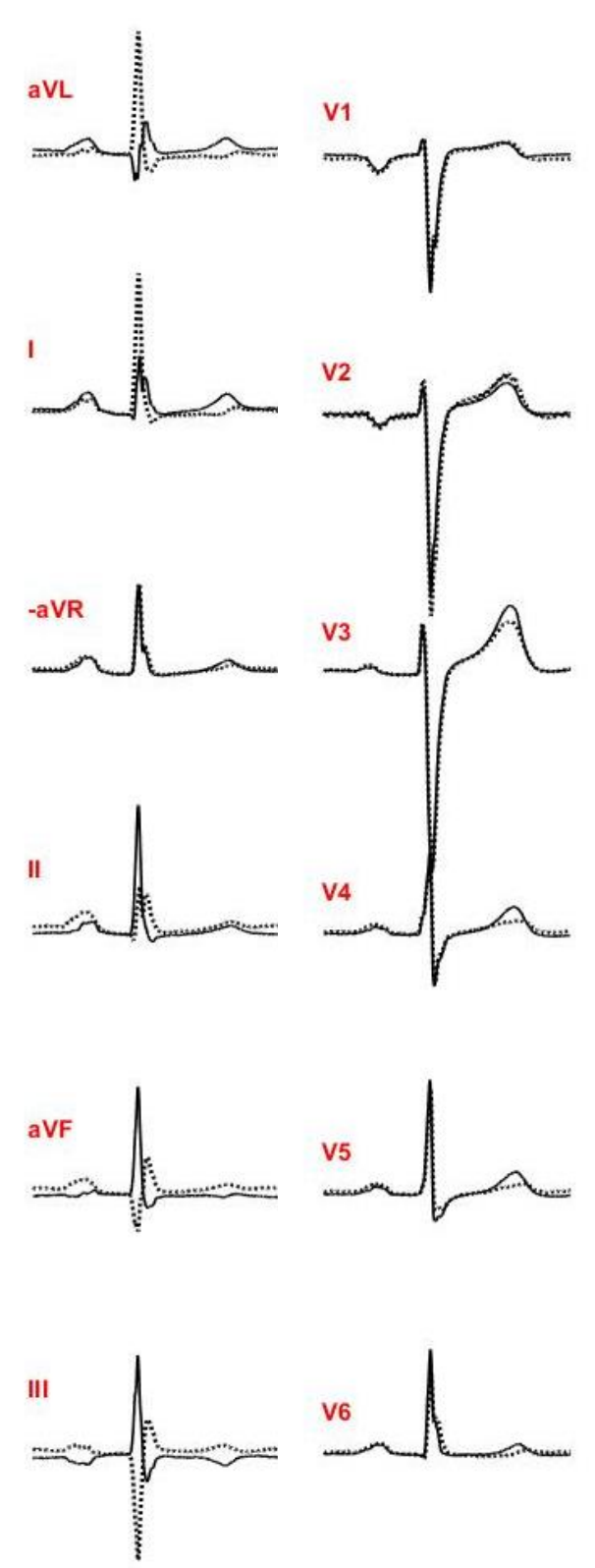

Figure 1. Average beat from the current (solid) and previous (dotted) ECGs with limb leads in Cabrera order. From the chest leads current and previous are very similar. The difference in the limb leads is due to a simulated LALL lead-wire interchange

in the previous ECG. When there are no significant clinical changes from previous ECG to the current ECG, the residual after subtraction is usually small. Figure 2 illustrates how well previous and current ECGs match across the study database by calculating the ratio of rootmean-square (RMS) difference divided by average RMS. With an LA-LL interchange, the difference in limb leads is in the same order of magnitude as the original signal while the precordial leads are unaffected and the precordial lead 
residual is still small. For ease of use with machine learning tools, the average beat residual was converted to tall narrow images, 12 leads wide and 300 samples tall at 500 sample/sec.

A simple VGG style convolutional neural network [10] was designed and trained to classify LA-LL interchange with an image input and a single output. The Cabrera lead order was used to keep highly correlated leads adjacent. Three convolutional layers were employed, each with a "relu" activation, batch normalization and drop out. Next came a 1000 neuron fully connected layer, "relu" activation, batch normalization, drop out and a final single neuron layer with sigmoid activation to generate the LALL output.

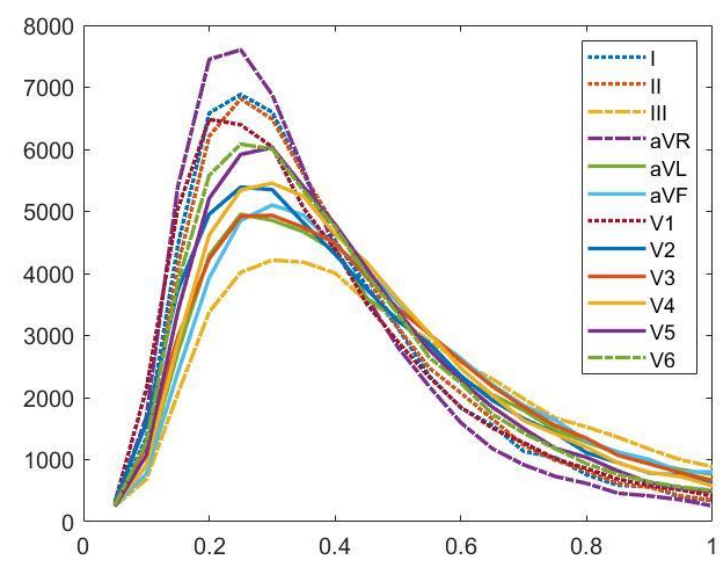

Figure 2. Histogram of how well current and previous ECG average beats match by lead. Quality of match is given the ratio of RMS difference (current - previous) divided by RMS average. The peaks near 0.3 mean that commonly the RMS voltage of the previous/current difference is $30 \%$ of the RMS voltage of the current average beat.

\subsection{Testing}

The algorithm performance was tested using sensitivity, specificity and estimated positive predictive value. The positive predictive value was estimated because the true prevalence is a fraction of $1 \%$ while the training prevalence was $50 \%$.

\section{Results}

The CNN algorithm detected the LA-LL interchange on the hold out test set with a sensitivity of $84.5 \%$ and a specificity of $99.5 \%$. At the expected prevalence of $0.5 \%$, the estimated positive predictive value and F1 score were $46 \%$ and $60 \%$ respectively.

\section{Discussion}

The algorithm presented here is the first to detect leadwire interchange based on serial ECG. Although it is a first for an algorithm, verification of lead reversal by serial ECG is common in manual ECG reading. In database development for lead-wire reversal detection, verification of interchange or lack of interchange is done by serial ECG comparison. Haar used serial ECGs to detect acute myocardial infarction by subtracting features calculated from previous and current ECG average beats [11]. Sbrollini used the same method to detect heart failure in post-infarction patients [12]. In both cases, a neural network was used to implement a classifier based on the difference features.

This is not the first published article to train a neural network to detect lead reversals that can be simulated. Hedén et.al. wrote two papers to introduce the use of neural networks for lead reversal detection [1] [7]. There are several differences comparing the present work to Hedén's. First, Hedén's network used well known ECG features as input from a single ECG. The present study uses images created directly from the waveforms, rather than features. Second, we use serial ECGs. Third, the present work takes advantage of deep learning neural networks which were not available at the time Hedén's papers were published. Finally, Hedén did not publish the performance of the LA-LL interchange. In earlier papers, our group also did not publish results for LA-LL because the performance was mediocre and therefore not worth attempting in clinical practice.

Kors published a paper on a wholly different method exploiting the redundancy in ECG signals [6]. The Kors algorithm detected the LA-LL interchange with a sensitivity and specificity of $18 \%$ and $99.5 \%$ respectively which was much lower than the detection performance of other lead-wire interchanges. Other lead wire interchanges had sensitivities in the 90s. The present work dramatically improves the LA-LL interchange compared to the Kors

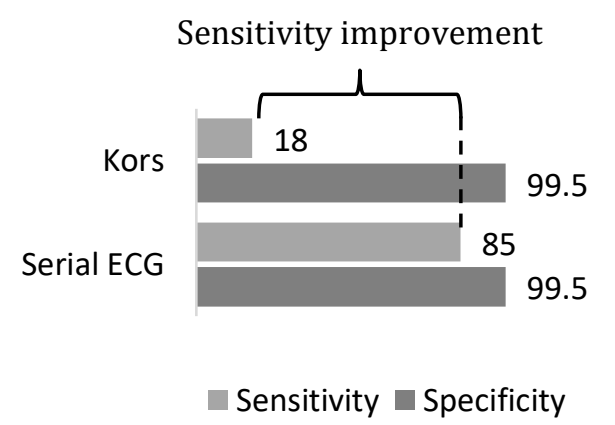

Figure 3. Comparison of the serial ECG and Kors algorithm performance for LA-LL lead-wire interchange in terms of sensitivity (lighter gray) and specificity (darker gray). 
algorithm as can be seen in Figure 3, but with the cost of adding the requirement of a previous ECG.

The serial ECG method is limited by availability of a previous ECG, however, a record of previous ECGs is common for patients with cardiac complaints [13]. The method is also limited by occurrence of new ECG changes expressed in the limb leads. As long as the clinical change is expressed in the chest leads as well at a level above day to day variation, the algorithm can presumably limit false positives because the CNN has learned normal chest lead variation. This is also an argument for a more complex machine learning model. The model must learn the difference between normal variation, change due to lead reversal in addition to health condition change. Finally, the impact of clinical change or time between previous and current ECGs on LA-LL interchange detection was not quantified. On the other hand, no selection was performed to remove ECGs representing a clinical change.

\section{Conclusion}

Detecting LA-LL lead-wire interchange is the most challenging in common lead-wire interchanges. Most algorithms for lead-wire interchange neglect the LA-LL case. Compared to the Kors algorithm with SE/SP of $17.5 / 99.5 \%$ for LA-LL interchange, this algorithm shows a significant boost in sensitivity (18\% to $85 \%)$ with the same specificity. The downside of the serial ECG based algorithm is the requirement for a previous ECG. However, viewing the previous ECG is common practice when manually interpreting ECG.

\section{References}

[1] B. Hedén, M. Ohlsson, L. Edenbrandt, R. Rittner, O. Pahlm and C. Peterson, "Artificial neural networks for recognition of electrocardiographic lead reversal," Am J Cardiol, vol. 75, pp. 929-33, 1995.

[2] J. C. Greenfield, "A bad day for quality control: sequential misplacement of left arm and left leg electrodes," J Electrocardiol, vol. 41, pp. 1-3, 2008.

[3] B. Bailey, R. E. Gregg and S. Babaeizadeh, "Influence of left arm and left leg ECG electrode reversal on lateral and inferior STEMI detection," J Electrocardiol, vol. 57, p. S98, 2019.

[4] S. Karur, S. Patra, R. K. Shankarappa, N. Agrawal and M. C. Nanjappa, Left arm-left leg lead reversal in a case of inferior wall myocardial infarction mimics as high lateral wall infarction," J Cardiovasc Disease Research, vol. 4, pp. 201-3, 2013.
[5] D. Q. Feild and R. Gregg, "Detecting lead reversals using the EASI to 12-lead electrocardiogram," J Electrocardiol, vol. 39, p. S129, 2006.

[6] J. A. Kors and G. van Herpen, "Accurate automatic detection of electode interchange in the electrocardiogram," Am J Cardiol, vol. 88, pp. 396-9, 2001.

[7] B. Hedén, M. Ohlsson, H. Holst, M. Mjijman, R. Rittner, O. Pahlm, C. Peterson and L. Edenbrandt, "Detection of frequently overlooked electrocardiographic lead reversals using artificial neural networks," Am J Cardiol, vol. 78, pp. 600-4, 1996.

[8] C. Han, R. E. Gregg, D. Q. Feild and S. Babaeizadeh, "Automatic detection of ECG cable interchange by analyzing both morphology and interlead relations," J Electrocardiol, vol. 47, no. 6, pp. 781-7, 2014.

[9] M. Hodges, D. Salerno and D. Erlien, "Bazett QT correction reviewed - evidence that a linear QT correction for heart-rate is better," JACC, vol. 1, no. 2, pp. 694-694, 1983.

[10] K. Simonyan and A. Zisserman, "Very deep convolutional networks for large-scale image recognition," arXiv, vol. 1409.1556, 2014.

[11] C. C. ter Haar, R. J. G. Peters, J. Bosch, C. A. Swenne et al, "An initial exploration of subtraction electrocardiography to detect myocardial ischemia in the prehospital setting," Ann Noninvasive Electrocardiol, vol. 25, p. e12722, 2020.

[12] A. Sbrollini1, M. C. De Jongh, C. Ter Haar, R. W. Treskes, S. Man, L. Burattini and C. A. Swenne, "Serial electrocardiography to detect newly emerging or aggravating cardiac pathology: a deep learning approach," BioMed Eng OnLine, vol. 18, no. 15, pp. 2-17, 2019.

[13] T. H. Lee, E. F. Cook, M. C. Weisberg, G. W. Rouan, D. A. Brand and L. Goldman, "Impact of the availability of a prior electrocardiogram on the triage of the patient with acute chest pain," J Gen Intern Med, vol. 5, pp. 381-8, 1990.

Address for correspondence:

Richard E. Gregg

222 Jacobs Street

Cambridge, MA 02141

rich.gregg@philips.com 